\title{
Modern contraceptive utilization and its associated factors among married women in Senegal: a multilevel analysis
}

Betregiorgis Zegeye ${ }^{1}$, Bright Opoku Ahinkorah², Dina Idriss-Wheeler ${ }^{3}$, Comfort Z. Olorunsaiye ${ }^{4}$, Nicholas Kofi Adjei ${ }^{5}$ and Sanni Yaya ${ }^{6,7^{*}}$ (1)

\begin{abstract}
Background: Utilization of modern contraceptives is still low in low-and middle-income countries, although fertility and population growth rates are high. In Senegal, modern contraceptive utilization is low, with few studies focusing on its associated factors. This study examined modern contraceptive use and its associated factors among married women in Senegal.

Methods: Data from the 2017 Continuous Demographic and Health Survey (C-DHS) on 11,394 married women was analysed. We examined the associations between the demographic and socioeconomic characteristics of women and their partners and modern contraceptive use using multilevel logistic regression models. Adjusted odds ratios with 95\% confidence intervals (Cl) were estimated.

Results: The utilization of modern contraceptives among married women was $26.3 \%$. Individual level factors associated with modern contraceptive use were women's age (45-49years-aOR $=0.44,0.30-0.63)$, women's educational level (higher-aOR $=1.88,1.28-2.76$ ) husband's educational level (higher-aOR $=1.43,1.10-1.85)$ ), number of living children ( 5 or more children-aOR $=33.14,19.20-57.22$ ), ideal number of children ( 2 children-aOR $=1.95,1.13-$ 3.35 ), desire to have more children (wants no more-aOR $=2.46,2.06-2.94$ ), ethnicity (Diola-aOR $=0.70,0.50-0.99$ ), media exposure (yes-aOR $=1.44,1.16-1.79)$ ), wealth index (richer-aOR $=1.31,1.03-1.67$ ) and decision making power of women (decision making two-aOR $=1.20,1.02-1.41$ ). Whereas, region (Matam-aOR $=0.35,0.23-0.53$ ), place of residence (rural$\mathrm{aOR}=0.76,0.63-0.93$ ), community literacy level (high-aOR $=1.31,1.01-1.71$ ) and community knowledge level of modern contraceptives (high-aOR $=1.37,1.13-1.67$ ) were found as significant community level factors.

Conclusions: The findings indicate that both individual and community level factors are significantly associated with modern contraceptive use among married women in Senegal. Interventions should focus on enhancing literacy levels of women, their husbands and communities. Furthermore, strengthening awareness and attitude towards family planning should be given priority, especially in rural areas and regions with low resources.
\end{abstract}

Keywords: Contraceptive utilization, Modern contraceptive, Senegal; sexual and reproductive health

\footnotetext{
* Correspondence: sanni.yaya@uOttawa.ca

${ }^{6}$ School of International Development and Global Studies, University of Ottawa, 120 University Private, Ottawa, ON K1N 6N5, Canada

${ }^{7}$ The George Institute for Global Health, Imperial College London, London, UK

Full list of author information is available at the end of the article
}

(c) The Author(s). 2021 Open Access This article is licensed under a Creative Commons Attribution 4.0 International License, which permits use, sharing, adaptation, distribution and reproduction in any medium or format, as long as you give appropriate credit to the original author(s) and the source, provide a link to the Creative Commons licence, and indicate if changes were made. The images or other third party material in this article are included in the article's Creative Commons licence, unless indicated otherwise in a credit line to the material. If material is not included in the article's Creative Commons licence and your intended use is not permitted by statutory regulation or exceeds the permitted use, you will need to obtain permission directly from the copyright holder. To view a copy of this licence, visit http://creativecommons.org/licenses/by/4.0/. The Creative Commons Public Domain Dedication waiver (http://creativecommons.org/publicdomain/zero/1.0/) applies to the data made available in this article, unless otherwise stated in a credit line to the data. 


\section{Background}

Family planning has been associated with several benefits such as reduction in maternal and infant mortality [1-4]. This can generally be achieved by encouraging girls' education [2, 4], and enhancing female labor force participation [4-7]. In developing countries, contraceptive use prevented 218 million unintended pregnnacies in 2012, averting 55 million unplanned births, 138 million abortions (of which 40 million were unsafe), 25 million miscariages and 118,000 maternal deaths [2]. In 2017, about $63 \%$ of married women of reproductive age or their partners used either modern or traditional methods of contraception $[5,8]$. However, compared to other continents, there is low prevalence of contraceptive use in Africa (36\%). For example, in 2017, contraceptive prevalence was $58 \%$ in Oceania while a prevalence of $75 \%$ was recorded in the Caribbean, Northern and Latin America $[5,8]$. Although there have been improvements in the use of contraceptives from $8 \%$ in 1970 to $36 \%$ in 2017 in Africa, there are still significant gaps in relation to the utilization of modern contraceptives among couples who have the intention to delay or prevent pregnancy $[5,8]$.

Senegal is a one of the African countries with a very high fertility rate and rapid population growth $[9,10]$. There are variations in total fertility rate (TFR) from 6.35 children per woman in rural areas to 3.9 per woman in urban areas [7]. Senegal is also one of the countries with lowest family planning utilization of $28 \%$ as at 2017 [5, 8]. Higher proportion of women are not getting family planning services, as the unmet need is estimated to be $29 \%$. That means, approximately one married women of every three women who has the intention to stop giving birth or delay her subsequent pregnancy is not using contraceptive. Unmet need was also higher (32\%) in Dakar region, where 25\% of the country's population live $[7,11]$.

There have been several studies on factors that influence contraceptive utilization in Africa [6, 12-19]. These studies have identified several individual and community-level factors such as age, marital status, ethnicity, religion, educational level, wealth index, place of residence and region as factors associated with moden contraceptive use [6, 12-19]. In terms of education, studies have shown that utilization of modern contraceptives is connected to partner's educational level $[13,20]$. Other studies suggest that husband's educational level may influence contraceptive use for family planning. Closely connected to education are findings that increased communication between couples facilitate support of contraceptive utilization by a husband $[20,21]$. Other studies have found associations between media exposure and contraceptive use [22]. The media has been found as beneficial in raising awareness on health issues within communities, as well as addressing social and cultural issues [23]. In terms of wealth, studies have found that the odds of contraceptive use is higher among richer women as compared to poorest [24-26] and this has been linked to the influence of socioeconomic status $[25,26]$. Married women with living children have been found to have higher odds of modern contraceptive use compared to married women with no living children $[6,27,28]$ similar to those who live in urban areas $[17$, 29]. The association between ethnicity and contraceptive utilization [30] has been attributed to unequal access of public services to people lower in the ethnic hierarchy, illiteracy, and poor socio-economic conditions among some ethnic groups [31-33].

In Senegal, despite the low contraceptive utilization, few studies have focused on factors associated with the low contraceptive usage [13, 17, 34-36]. Moreover, available studies on utilization of contraceptivs were either based on the 2012/13 DHS [35] and 1992-2013/14 DHS data [13], focused on single factor [13, 34] and limited to a single study area $[34,36]$. None of these studies have examined the association between individual and community-level factors and utilization of contraceptives in Senegal. Therefore, the present study aimed to examine the prevalence and factors associated with the use of modern contraceptives among married women in Senegal.

The findings of the study will inform existing policies aimed at enhancing the use of contraceptives in Senegal. Among the important policies in Senegal is the $2005 \mathrm{Re}$ productive Health $(\mathrm{RH})$ Law, which is based on the right of individuals and couples to $\mathrm{RH}$, equitable access to $\mathrm{RH}$ care, and respect for the physical integrity of women and girls $[37,38]$. In addition, giving priorities for disadvantaged women or narrowing of disparities in the use of contraceptive is another strategy that exists in Senegal and the findings of the current study will influence the success of these strategies. Finally, the findings of the current study will also help to enhance the strategy of ensuring increase service uptake of contraceptive in Senegal through media exposure [39].

\section{Methods \\ Study setting}

Senegal, located in West Africa, is well-known as the "Entry to Africa" [40, 41]. Up to half of its 15.4 million people (2016) live in and around Dakar and other urban areas [40]. In Senegal, there is a hierarchical health system model, where regional medical officers oversee the activities of health districts in every region. Health districts are made up of a health centre and a linkage to rural health posts, often under the supervision of allied community-level facilities [42]. These health facilties are operated by community health workers [43]. In senegal, family planning usage has been low- only about $18 \%$ of women with partners aged 15-49 used a modern 
contraceptive method in 2013 [10]. In 2015, it increased marginally to $21.2 \%$, and $21-23 \%$ had an unmet neednon-use of contraceptives among fecund and sexually active women who do not want any more pregnancy or have the intention to delay the next pregnancy [44], for family planning [45].

\section{Data source}

Data from the 2017 Continuous-Demographic and Health Survey (C-DHS) [45] was used in this study. The C-DHS is a nationally representative data undertaken by the Senegal Agency of Statistics and Demography (ANSD) in collaboration with ICF International [45]. A two-stage stratified sampling procedure is adopted in selecting research participants. In the first phase, clusters/enumeration areas (EAs) are selected, guided by a sample frame developed during the 2013 census [45]. In all, 8800 households, made up of 4092 located in urban areas and 4708 in rural areas were considered in the survey [45]. For this study, a sample of 11,394 married women aged 15-49 with information on modern contraceptive use were considered.

\section{Variables}

The main outcome variable of this study was modern contraceptive use. To derive this variable, married women were asked of the contraceptive methods they use. Those who mentioned that they used modern contraceptives were coded as modern contraceptive method users while those who used traditional/folkloric/no method were coded as non-modern contraceptive users. Details of the types of contraceptives considered as modern, traditional or folkloric have been published in previous studies $[2,4,46]$. Married women who were sterilized and declared as infecund were excluded from the analysis. Several individual and community-level explanatory variables were chosen based on prior evidence $[6,14-16,18,19,36,47-50]$.

\section{Individual level factors}

These included demographic and socio-economic variables such as women's age (15-19, 20-24, 25-29, 30-34, $35-39,40-44,45-49)$ women's educational level (No formal education, primary school, secondary school, higher), husband/partner education (No formal education, primary school, secondary school, higher), number of living children (no children, 1-2 children, 3-4 children, five or more children), ideal number of children (no child, 1 child, 2 children, 3 children, 4 children, 5 children, 6 children and above and non-numeric response (e.g upto God/Allah) and desire for more children (wants within 2 years, wants after 2 years, wants but unsure of timing, undecided, wants no more). Others were distance to health facility (a big problem, not a big problem), ethnicity (Wolof, Poular, Serer, Mandingue/ soc., Diola, Sonink, other Senegalese and other), religion (Muslim and Christian) and media exposure (newspaper, radio or television [TV]). Media exposure was derived from a combination of three variables; reading of newspaper or magazine, listening to radio and watching television. DHS grouped all of the three variables into three categories not at all (0), less than once a week (1) and at least once a week (2). We then, re-categorized these into two (binary) for all the three variables. Those who didn't read newspaper/magazine, or listened to radio or watched television at all were coded as zero (0), and those who read newspaper/magazine, listened to radio or watched television for less than once a week and at least once a week were coded as one (1). We then generated, media exposure by adding the three variables. If the respondent had no exposure at all for all three variables we coded as 'no'. If the respondent had exposure to at least one of the three variables, we coded as 'yes'.

Other individual level variables included wealth index (poorest, poorer, middle, richer, richest), attitude towards wife beating (refuse versus accept) and women's empowerment $[51,52]$. Women empowerment as a variables was derived from respondent's decision making capacity on own health care, large household purchases, and visits to family and relatives. Respondents whose responses to these three decision making variables showed that their decisions are made by their husband/partner alone or other were considered as having "no decisionmaking". Respondents who mentioned that they took decisions alone or with their husband/partner on two of the decision making variables were put in the category "decision making one". Finally, women who decided alone or with their partner/husband on all the three decision making variables were coded into "decision making two".

\section{Community level factors}

Place of residence (urban, rural), region (Dakar, Ziguinchor, Diourbel, Saint Louis, Tambacounda, Kaolack, Thies, Louga, Fatick, Kolda, Matam, Kaffrine, Kedougo and Sedhiou), community-level literacy-proportion of women who can read and write (low medium, high), communitylevel socioeconomic status-the proportion of women within the richest wealth quintile (low, medium, high) and community-level knowledge about modern contraceptivethe proportion of women with knowledge about modern contraceptives (low, medium, high) were considered as community-level variables.

\section{Statistical analysis}

This study employed both descriptive statistics and multi-level logistic regression models in the data analyses. The descriptive analysis employed percentages to describe the study variables. This was followed by the 
use of chi-square test to show the independent associations between the individual and community level variables and modern contraceptive use using $p$-values < 0.05 as cut of points. Finally, multilevel logistic regression analysis made up of four models was carried out using the "melogit" stata command. Results were presented using adjusted odds ratios (aOR) at 95\% confidence intervals $(\mathrm{CI})$. The multilevel logistic regression models consisted of fixed effects (measures of association) and random effects (measures of variability) [19, 53, 54]. Muli-collinearity test was done among the independent variables using the variance inflation factor (VIF) and the results indicated no evidence of high collinearity among the explanatory variables (Mean VIF $=$ 1.43 , Minimum VIF $=1.01$, Maximum VIF $=2.26$ ). Previous studies have shown that VIF values less than 10 are tolerable [55-57]. Sample weights were employed and the 'svyset' command was used to correct for under and over-sampling $[26,58]$. All analyses were carried out in stata 14 for windows.

\section{Ethical consideration}

Data for this study was derived from the website of MEASURE DHS through a standard national survey. Permission to use the data was granted by MEASURE DHS review board. All data were anonymized prior to the authors receiving the data.

\section{Results}

\section{Background characteristics of respondents and use of modern contraceptives}

Out of the 11,394 married women, $26.3 \%$ were using modern contraceptives. Results on the distribution of modern contraceptive use across the explanatory variables show that most of the women who used modern contraceptives were aged 35-39 (29.62\%), had higher level of education (36.68\%), had husbands with higher level of education (34.83\%), lived in urban areas (31.36\%) and belonged to the Serer ethnic group (28.47\%). The results further showed that most of the women who used modern contraceptives were Christians (217.61\%), had richest wealth index (33.84\%), had five or more children (32.75\%), were against wife beating (27.62\%), lived in Dakar (42.05\%) and were exposed to media (24.19\%). Further results also indicate high modern contraceptive use among women with 2 children (34.23\%), those who considered distance to the health facility as not a big problem (25.13\%), those who lived in high literacy level communities (33.47\%), those who lived in communities with high socio-economic status (32.65\%) and those who lived in communities where knowledge of modern contraceptives was high (28.94\%). The results of the bivariate analysis indicate that all the explantatory variables had statistically significant associations with modern contraceptive use at $p<0.05$ (Table 1 ).

\section{Factors associated with modern contraceptive use}

The results on the individual level factors of modern contraceptive use showed that women's age (45-49 years$\mathrm{aOR}=0.44, \quad 0.30-0.63)$, women's educational level (higher-aOR $=1.88,1.28-2.76$ ) and husband's educational level (higher-aOR $=1.43,1.10-1.85$ ) were associated with modern contraceptive use among married women in Senegal. Number of living children ( 5 or more children$\mathrm{aOR}=36.01,22.73-57.05)$, ideal number of children (2 children-aOR $=1.95,1.13-3.35)$, desire to have more children (wants no more-aOR $=2.46,2.06-2.94$ ), ethnicity (Diola-aOR $=0.70,0.50-0.99$ ) and media exposure (yes$\mathrm{aOR}=1.44,1.16-1.79)$ also had significant associations with modern contraceptive use among married women in Senegal. Other individual level factors associated with modern contraceptive use were wealth index (richer$\mathrm{aOR}=1.31,1.03-1.67)$ and decision making power of women (decision making two-aOR $=1.20,1.02-1.41$ ). In relation to the community-level factors, region (Matam$\mathrm{aOR}=0.35,0.23-0.53$ ), place of residence (rural-aOR= $0.76,0.63-0.93$ ), community literacy level (high-aOR = $1.31,1.01-1.71$ ) and community knowledge level of modern contraceptive (high-aOR $=1.37,1.13-1.67$ ) were found as significant factors (see Model 3 of Table 2).

In terms of model fitness, the complete model (Model 3 ), which included all the individual and community level factors had the highest AIC of 10,226.48 and the lowest log-likelihood ratio of -5048.23 and was thus considered as the best fit model for predicting the occurrence of modern contraceptive use among married women.

\section{Discussion}

Our study examined modern contraceptive use and its associated factors among married women in Senegal. Age, women's and husband's level of education, women's decision making capacity, exposure to media, number of living children, desire to have more children, ideal number of children, place of residence, ethnicity, community literacy level, community level modern contraceptive knowledge and region were associated with modern contraceptive use.

In line with previous studies [47, 48], our findings showed that the use of modern contraceptive decreases with age, controlling for other factors. We observed that the use of modern contraceptives was high among younger women compared to older women. This higher uptake among younger women has been attributed to effective communication on family planning matters with their husbands/partners [49].

We found that women's educational level influences uptake of modern contraceptive use, with better utilization among women with higher educational level, 
Table 1 Utilization of modern contraceptives across explanatory variables Variables

\begin{tabular}{l} 
Modern Contraceptive use \\
\hline Women's age \\
$15-19$ \\
$20-24$ \\
$25-29$ \\
$30-34$ \\
$35-39$ \\
$40-44$ \\
$45-49$ \\
Women's educational leve \\
No formal education \\
Primary school \\
Secondary school \\
Higher
\end{tabular}

Husband's educational level

No formal education
Primary school
Secondary school
Higher

\section{Place of residence}

$$
\begin{aligned}
& \text { Urban } \\
& \text { Rural }
\end{aligned}
$$

\section{Ethnicity}

Wolof

Poular

Serer

Mandingue/soc

Diola

Sonink

Other Senegalese

Other

\section{Religion}

Muslim

Christian

\section{Wealth index}

Poorest

Poorer

Middle

Richer

Richest

\section{Number of living children}

No child

1-2 children

\begin{tabular}{l} 
Non use \\
Frequency/Percent \\
\hline $8770(73.69)$
\end{tabular}

Use

Chi-square, $P$-value

Frequency/Percent

2624 (26.31)

$\begin{array}{ll}963(92.15) & 82(7.85) \\ 1566(82.16) & 340(17.84) \\ 1706(76.26) & 531(23.74) \\ 1598(72.64) & 602(27.36) \\ 1181(70.38) & 497(29.62) \\ 1004(72.81) & 375(27.19) \\ 752(79.24) & 197(20.76)\end{array}$

$5820(79.79)$

$1736(72.12)$

1087 (72.81)

$126(63.32)$

6703 (80.15)

922 (66.71)

825 (71.24)

$320(65.17)$

2946 (68.64)

5824 (82.01)

2767 (74.91)

$3075(81.24)$

1045 (71.53)

798 (77.63)

$263(72.65)$

149 (80.11)

415 (73.19)

258 (82.96)

8555 (77.09)

215 (72.39)

$2572(82.94)$

2218 (81.97)

1934 (75.28)

1215 (68.96)

831 (66.16)

1380 (98.29)

2906 (79.79) $x 2=246.16, p<0.001$

$1474(20.21)$

671 (27.88)

406 (27.19)

73 (36.68)

$x 2=189.69, p<0.001$

1660 (19.85)

460 (33.29)

333 (28.76)

171 (34.83)

$X 2=100.18, p<0.001$

$x 2=269.61, p<0.001$

$1346(31.36)$

1278 (17.99)

$x 2=88.21, p<0.001$

927 (25.09)

710 (18.76)

416 (28.47)

230 (22.37)

99 (27.35)

37 (19.89)

152 (26.81)

53 (17.04)

$x^{2}=3.60, p=0.057$

2542 (22.91)

82 (27.61)

$X 2=251.21, p<0.001$

529 (17.06)

488 (18.03)

635 (24.72)

547 (31.04)

425 (33.84)

$X 2=525.33, p<0.001$
736 (20.21) 
Table 1 Utilization of modern contraceptives across explanatory variables Variables (Continued)

\section{Modern Contraceptive use \\ 3-4 children \\ Five or more children \\ Decision making \\ No decision making \\ Decision making one \\ Decision making two \\ Wife-beating}

Accept

Refuse

Region

Dakar

Ziguinchor

Diourbel

Saint Louis

Tambacounda

Kaolack

This

Louga

Fatick

Kolda

Matam

Kaffrine

Kedougou

Sedhiou

\section{Media exposure}

No

Yes

Desire for more children

Wants within 2 years

Wants after 2 years

Wants, unsure timing

Undecided

Does not want

Ideal number of children

0 child

1 child

2 children

3 children

4 children

5 children

6 children and above

Non-numeric response

\section{Non use}

Frequency/Percent

2212 (71.89)

$2272(69.46)$

$5918(80.87)$

1969 (71.26)

$883(67.25)$

5361 (80.21)

3409 (72.38)

481 (57.95)

$352(71.69)$

$813(83.30)$

$563(73.02)$

$753(85.67)$

$559(76.26)$

615 (66.13)

$694(78.77)$

$617(72.59)$

$683(78.51)$

$771(89.24)$

766 (77.45)

$520(88.29)$

$583(78.68)$

964 (87.88)

7806 (75.81)

2898 (87.98)

3380 (71.93)

705 (89.92)

141 (79.66)

1379 (64.71)

164 (73.54)

$12(80.00)$

98 (65.77)

346 (75.22)

1199 (71.84)

1414 (73.49)

3811 (77.35)

$1726(85.15)$
Use

Frequency/Percent

865 (28.11)

999 (30.54)

1400 (19.13)

794 (28.74)

430 (32.75)

$x 2=95.52, p<0.001$

1323 (19.79)

1301 (27.62)

$x^{2}=434.48, p<0.001$

349 (42.05)

139 (28.31)

163 (16.70)

208 (26.98)

126 (14.33)

174 (23.74)

315 (33.87)

187 (21.23)

$233(27.41)$

187 (21.49)

$93(10.76)$

223 (22.55)

69 (11.71)

158 (21.32)

133 (12.12)

$2491(24.19)$

$\times 2=543.34, p<0.001$

396 (12.02)

1319 (28.07)

79 (10.08)

36 (20.34)

752 (35.29)

$X 2=127.72, p<0.001$

59 (26.46)

$3(20.00)$

51 (34.23)

114 (24.78)

470 (28.16)

510 (26.51)

$1116(22.65)$

301 (14.85) $x 2=81.44, p<0.001$ 
Table 1 Utilization of modern contraceptives across explanatory variables Variables (Continued)

\begin{tabular}{|c|c|c|c|}
\hline \multirow[t]{2}{*}{ Modern Contraceptive use } & \multirow{2}{*}{$\begin{array}{l}\text { Non use } \\
\text { Frequency/Percent }\end{array}$} & Use & Chi-square, $P$-value \\
\hline & & \multicolumn{2}{|l|}{ Frequency/Percent } \\
\hline Distance to health facility & & & $x^{2}=65.37, p<0.001$ \\
\hline Big problem & $2824(81.81)$ & $628(18.19)$ & \\
\hline Not a big problem & $5946(74.87)$ & $1996(25.13)$ & \\
\hline Community litracy level & & & $x 2=348.26, p<0.001$ \\
\hline Low & $3972(84.78)$ & $713(15.22)$ & \\
\hline Medium & $2826(75.46)$ & 919 (24.54) & \\
\hline High & $1972(66.53)$ & $992(33.47)$ & \\
\hline Community socioeconomic level & & & $x^{2}=258.56, p<0.001$ \\
\hline Low & $5477(81.92)$ & $1209(18.08)$ & \\
\hline Medium & $1191(75.05)$ & $396(24.95)$ & \\
\hline High & $2102(67.35)$ & $1019(32.65)$ & \\
\hline \multicolumn{4}{|c|}{ Community level modern contraceptive knowledge } \\
\hline Low & $3391(84.00)$ & $646(16.00)$ & $x 2=190.92, p<0.001$ \\
\hline Medium & $2820(75.08)$ & 936 (24.92) & \\
\hline High & $2559(71.06)$ & $1042(28.94)$ & \\
\hline
\end{tabular}

which is supported by a previous study [49]. As the level of education increases, wealth and prestige tend to increase and the intention to limit children by using modern contraceptives will increase [50]. Apparently, education leads to a greater ability to acquire wealth and prestige; this competes with decision on childbearing since in a modern economy children are for many years resource consumers rather than resource producers [50]. The findings indicate the need to consider education during awareness creation such as using audio-visual aid instead of leaflets distribution.

The study further revealed that utilization of modern contraceptives is connected to partner's educational level, and media exposure. The odds of use of modern contraceptives among married women whose partners had completed at least primary education were higher compared to those whose partners had no formal education. This is consistent with the findings of previous studies $[13,20]$ that have shown that partners/husbands with higher levels of education may support women's contraceptive usage through high levels od communication $[20,21]$. Relatedly, women who had exposure to newspaper, radio or television for at least once a week had higher likelihood of using modern contraceptive methods than women with no media exposure. Mass media has been considered as a powerful tool that can increase the demand for health services as it provides individuals within a community the means to identify, respond to, and address their own needs [22]. The media may also be beneficial in raising awareness on health issues within communities, as well as addressing social and cultural issues. This crucial role of the media can help reduce the barriers to access and use of health services including contraceptive services [23].

Consistent with findings from prior studies [24-26], we further found that the odds of modern contraceptive use was higher among richer women as compared to poorest. This could be due to wealth influence, related to their socioeconomic status including access to modern health care and education $[25,26]$. We further found a significant association between the number of living children and the use of modern contraceptive, with married women with living children more likely to use modern contraceptives compared to those with no living children. Similar findings have been obtained in previous studies [6, 27, 28].

Living in urban areas was found to lead to a higher likelihood of using modern contraceptive methods than in rural areas, consistent with previous studies [17, 29]. This could be due to the fact that urban residents are less likely to travel long distances for medical services than their rural counterparts [59]. Furthermore, not all health facilities are equipped with contraceptive methods, particularly in Southern regions [59, 60]. According to previous studies, a national intervention aimed at strengthening the contraceptive supply chain had not been implemented in the southern part of the country [17, 60]. Available evidence in Senegal [17] suggested that knowledge about contraceptive methods is highly associated with uptake and it has been shown that those living in rural areas may have little knowledge on modern contraceptive methods [17]. In Senegal, low contraceptive demand among rural women may be due to geographical and financial barriers associated with 
Table 2 Multilevel binary logistic regression results for factors associated with modern contraceptive use among married women in Senegal

\begin{tabular}{l} 
Variables \\
\hline Decision making \\
Decision making one \\
Decision making two \\
Wife-beating \\
Accept (ref) \\
Refuse \\
Media exposure \\
No (ref) \\
Yes \\
Women's educational level \\
No formal education (ref) \\
Primary school \\
Secondary school \\
Higher
\end{tabular}

\section{Religion}

Muslim (ref)

Christian

$$
\begin{aligned}
& 1.24(1.10-1.40)^{* * *} \\
& 1.28(1.09-1.50)^{* *}
\end{aligned}
$$

$1.13(1.01-1.26)^{*}$

$1.11(0.99-1.24)$

$1.54(1.24-1.91)^{* * *}$

$1.44(1.16-1.79)^{* *}$

$1.24(1.09-1.42)^{* *}$

$1.42(1.19-1.70)^{* * *}$

$1.98(1.34-2.92)^{* * *}$

$1.08(0.78-1.50)$

\section{Wealth index}

Poorest (ref)

Poorer

Middle

Richer

Richest

\section{Women's age}

15-19years (ref)

20-24 years

25-29years

30-34 years

35-39 years

40-44 years

45-49 years

Number of living children

No child (ref)

1-2 children

3-4 children

5 or more children

\section{Ethnicity}

Wolof (ref)

Poular

$0.87(0.75-1.01)$

Serer

Mandingue/soc

Diola
1.01 (0.86-1.19)

$1.41(1.18-1.69)^{* * *}$

$1.72(1.40-2.11)^{* * *}$

$1.79(1.40-2.27)^{* * *}$

$1.18(0.88-1.57)$

$1.10(0.82-1.47)$

$0.98(0.72-1.33)$

$0.94(0.68-1.29)$

$0.70(0.50-0.98)^{*}$

$0.47(0.32-0.68)^{* * *}$

$12.60(8.23-19.30)^{* * *}$

$24.47(15.72-38.08)^{* * *}$

$35.49(22.38-56.27)^{* * *}$
$1.03(0.75-1.43)$

$1.21(1.06-1.38)^{* *}$
$1.37(1.14-1.64)^{* * *}$
$1.88(1.28-2.76)^{* *}$

$0.97(0.82-1.14)$

$1.20(0.99-1.46)$

$1.31(1.03-1.67)^{*}$

$1.22(0.91-1.62)$

$1.15(0.87-1.54)$

$1.05(0.78-1.40)$

$0.94(0.69-1.28)$

$0.88(0.64-1.22)$

$0.66(0.47-0.92)^{*}$

$0.44(0.30-0.63)^{* * *}$

$12.55(8.20-19.21)^{* * *}$

$24.51(15.76-38.11)^{* * *}$ $36.01(22.73-57.05)^{* * *}$

$0.90(0.77-1.06)$

1.08 (0.90-1.31)

$1.04(0.82-1.31)$

$0.70(0.50-0.99)^{*}$
$1.10(0.91-1.32)$

$0.76(0.55-1.05)$ 
Table 2 Multilevel binary logistic regression results for factors associated with modern contraceptive use among married women in Senegal (Continued)

\begin{tabular}{l} 
Variables \\
\hline Sonink \\
Other Senegalese \\
Other \\
Husband educational level \\
No formal education (ref) \\
Primary school \\
Secondary school \\
Higher
\end{tabular}

\section{Ideal number of children}

0 child (ref)
1 child
2 children
3 children
4 children
5 children
6 children and above
Non-numeric response

$$
\begin{aligned}
& 0.79(0.18-3.41) \\
& 1.85(1.07-3.17)^{*} \\
& 1.07(0.70-1.66) \\
& 1.17(0.80-1.70) \\
& 1.22(0.84-1.77) \\
& 1.06(0.74-1.52) \\
& 0.80(0.55-1.18)
\end{aligned}
$$

Model III

$0.99(0.62-1.57)$

$0.96(0.74-1.23)$

$0.66(0.46-0.95)^{*}$

Desire for more children

Wants within 2 years (ref)

Wants after 2 years

Wants, unsure timing

Undecided

Wants no more

\section{Distance to health facility}

Big problem (ref)

Not a big problem

\section{Region}

Dakar (ref)

Ziguinchor

Diourbel

Saint Louis

Tambacounda

Kaolack

Thies

Louga

Fatick

Kolda

Matam

Kaffrine

Kedougou

Sedhiou

$\begin{array}{ll}0.60(0.42-0.86)^{* *} & 0.69(0.46-1.04) \\ 0.50(0.35-0.69)^{* * *} & 0.47(0.33-0.67)^{* * *} \\ 0.73(0.53-1.00) & 0.81(0.57-1.14) \\ 0.56(0.39-0.81)^{* *} & 0.77(0.51-1.16) \\ 0.63(0.46-0.87)^{* *} & 0.70(0.50-1.00) \\ 0.99(0.74-1.32) & 0.96(0.70-1.32) \\ 0.67(0.48-0.93)^{*} & 0.75(0.52-1.07) \\ 0.82(0.59-1.14) & 0.75(0.53-1.08) \\ 0.67(0.48-0.93)^{*} & 0.85(0.59-1.23) \\ 0.30(0.21-0.44)^{* * *} & 0.35(0.23-0.53)^{* * *} \\ 0.86(0.61-1.20) & 1.04(0.72-1.51) \\ 0.37(0.25-0.57)^{* * *} & 0.51(0.32-0.80)^{* *} \\ 0.60(0.43-0.86)^{* *} & 0.70(0.48-1.03)\end{array}$

$1.10(0.97-1.26)$

$2.09(1.81-2.40)^{* * *}$

$0.93(0.70-1.23)$

$1.52(0.99-2.32)$

$2.46(2.06-2.94)^{* * *}$ 
Table 2 Multilevel binary logistic regression results for factors associated with modern contraceptive use among married women in Senegal (Continued)

\begin{tabular}{|c|c|c|c|c|}
\hline Variables & Model 0 & Model I & Model II & Model III \\
\hline \multicolumn{5}{|l|}{ Place of residence } \\
\hline \multicolumn{5}{|l|}{ Urban (ref) } \\
\hline Rural & & & $0.76(0.63-0.91)^{* *}$ & $0.76(0.63-0.93)^{* *}$ \\
\hline \multicolumn{5}{|l|}{ Community literacy level } \\
\hline \multicolumn{5}{|l|}{ Low (ref) } \\
\hline Medium & & & $1.47(1.22-1.78)^{* * *}$ & $1.30(1.07-1.59)^{* *}$ \\
\hline High & & & $1.65(1.29-2.120)^{* * *}$ & $1.31(1.01-1.71)^{*}$ \\
\hline \multicolumn{5}{|c|}{ Community socioeconomic level } \\
\hline \multicolumn{5}{|l|}{ Low (ref) } \\
\hline Medium & & & $1.15(0.93-1.42)$ & $1.08(0.86-1.36)$ \\
\hline High & & & $1.10(0.88-1.37)$ & $1.04(0.81-1.33)$ \\
\hline \multicolumn{5}{|c|}{ Community level modern contraceptive knowledge } \\
\hline \multicolumn{5}{|l|}{ Low (ref) } \\
\hline Medium & & & $1.37(1.15-1.62)^{* * *}$ & $1.34(1.12-1.61)^{* * *}$ \\
\hline High & & & $1.46(1.22-1.76)^{* * *}$ & $1.37(1.13-1.67)^{* * *}$ \\
\hline \multicolumn{5}{|l|}{ Random effect result } \\
\hline PSU variance $(95 \% \mathrm{Cl})$ & $0.52(0.42-0.65)$ & $0.30(0.23-0.40)$ & $0.16(0.11-0.22)$ & $0.16(0.11-0.24)$ \\
\hline ICC & 0.13 & 0.08 & 0.04 & 0.04 \\
\hline LR Test & $X^{2}=443.55, p<0.001$ & $X^{2}=152.76, p<0.001$ & $x^{2}=76.02, p<0.001$ & $X^{2}=59.26, p<0.001$ \\
\hline Wald chi-square and p-value & & $X^{2}=883.97, p<0.001$ & $X^{2}=343.82, p<0.001$ & $X^{2}=1028.03, p<0.001$ \\
\hline \multicolumn{5}{|l|}{ Model fitness } \\
\hline Log-likelihood & -5926.8 & -5113.3 & -5793.98 & -5048.23 \\
\hline AlC & $11,857.64$ & $10,316.66$ & $11,631.96$ & $10,226.48$ \\
\hline $\mathrm{BIC}$ & $11,872.32$ & $10,645.76$ & $11,793.46$ & $10,701.84$ \\
\hline PSU & 400 & 400 & 400 & 400 \\
\hline
\end{tabular}

Notes: AOR Adjusted odds ratios, COR Crude odds ratio ${ }^{* * *} p<0.001,{ }^{* *} p<0.01,{ }^{*} p<0.05$. Ref $=$ reference

access to contraceptive services [61], as well as reluctance to use family services [62].

Similarly, we observed differences in the utilization of modern contraceptive across regions, consistent with previous findings [17]. Modern contraceptives use was identified to be lower in almost all the regions as compared to Dakar region. This finding can be attributed to the existence of family planning services, and the increasing number of health facilities in urban areas [13]. Prior studies in Senegal noted an unequal repartition of health structures, and health personnel across regions with higher absorptions and accumulations of potentialities in few regions such as Dakar regions as major concern for creating disparities across regions $[13,17,35,60]$.

A significant association was found between ethnicity and contraceptive utilization as seen in a prior study [30]. This phenomenon may be attributed to the unequal access of public services to people lower in the ethnic hierarchy, illiteracy, and poor socio-economic conditions among some ethnic groups. Other reasons for the disparities in modern contraceptive use across ethnic groups could be the health insurance use, attitudes of physicians towards minority people, language barriers, transportation cost, low level of education, illiteracy, poverty and low socio-economic status, familiarity with the health care delivery system, the degree and kind of family support [31-33].

In line with a previous study [18], we found better uptake of modern contraceptives among married women with decision making power as compared to married women who had no decision making power. Beside increasing uptake of contraceptive methods, empowering women has an imperative role in the decrease of maternal and new born mortality by preventing unplanned pregnancy and unsafe abortion [18]. We also found that community-level literacy and community knowledge of modern contraceptives affect the utilization of modern contraceptive. Education can empower societies including women through improving their independence and involvement in decision-making, absolutely transforming 
health-seeking behavior and building social capital through development of social networks [59].

\section{Strengths and limitation of the study}

The key strength of this study lies in the use of nationally representative DHS data and a large sample size. Nonetheless, some study limitations were also observed. The study design (cross-sectional) does not give us the opportunity to establish causality between variables. Second, since we used secondary data, other determinants such as quality of care including counseling about side effects and accessibility of supply, and cultural factors were not included in the models. To deal with the limitations associated with the cross-sectional design and the absence of some essential variables in this study, a longitudinal study that uses primary data and gathers information on all relevant factors associated with the use of modern contraceptives is recommended.

\section{Conclusion}

Utilization of modern contraceptives may be influenced by both individual and community-level factors. While, women's age, women and husband's educational level, media exposure, number of living children, desire to have more children, ideal number of children, economic status, women decision making power, ethnicity were the individual-level factors, place of residence, community literacy level, community level modern contraceptive knowledge and subnational region were found to be the important community-level determinants for the utilization of modern contraceptive methods in this study. Therefore, interventions should focus on enhancing literacy levels, for both the women and their husbands. Additionally, to satisfay the demand of disadvantaged women (uneducated, rural residents, poor, and those living in some regions), using opportunities like mini media promotion campaign in a place where those women are available including market day could be considered. Moreover, integrating contraceptive service (offer) with child health service such as child Out Patient Department (OPD), immunization service, could be in fixed or mobile vaccination site as well as with maternal service such as couple counseling about contraceptive during ANC care and offering the service during PNC and post abortion time. Furthermore, strengthening awareness and attitude towards family planning should be given priority, in addition to designing intervention to increase accessibility and affordability of contraceptive methods especially in rural areas and regions with low resources. Finally, empowering women economically and through education can be helpful.

\section{Abbreviations}

ANC: Antenatal care; AOR: Adjusted Odd Ratio; Cl: Confidence Interval; C-

DHS: Continuous Demographic and Health Survey; COR: Crude Odd Ratio;
DHS: Demographic and Health Survey; EA: Enumeration Area; ICF: Inner City Fund; IR: Individual Recode; IRB: Institutional Review Board; PNC: Posnatal care; PPS: Probability Proportional to Size; PSU: Primary Sampling Unit; SDG: Sustainable Development Goal; WHO: World Health Organization

\section{Acknowledgments}

We acknowledge the Demographic and Health Surveys Program for making the DHS data available, and we thank the women who participated in the surveys.

\section{Authors' contributions}

SY and BZ contributed to the conception and design of the study, interpreted the data, prepared the manuscript, and led the paper. BOA, DIW, CZO and NKA helped with data analysis, provided technical support in interpretation of results and critically reviewed the manuscript for its intellectual content. All authors read and revised drafts of the paper and approved the final version.

Funding

No funding was received for this work.

\section{Availability of data and materials}

The datasets generated and/or analyzed during the current study are available in http://dhsprogram.com/data/available-datasets.cfm.

\section{Ethics approval and consent to participate}

Ethics approval was not required since the data is available to the public domain.

\section{Consent for publication}

Not applicable.

\section{Competing interests}

Sanni Yaya and Bright Opoku Ahinkorah are Editorial Board Members of BMC Public Health.

\section{Author details}

${ }^{1}$ HaSET Maternal and Child Health Research Program, Shewarobit Field Office, Shewarobit, Ethiopia. ${ }^{2}$ School of Public Health, Faculty of Health, University of Technology Sydney, Ultimo, Australia. ${ }^{3}$ Interdisciplinary School of Health Sciences, University of Ottawa, Ottawa, Ontario, Canada. ${ }^{4}$ Department of Public Health, Arcadia University, Glenside, PA, USA. ${ }^{5}$ Leibniz Institute for Prevention Research and Epidemiology, BIPS, Bremen, Germany. ${ }^{6}$ School of International Development and Global Studies, University of Ottawa, 120 University Private, Ottawa, ON K1N 6N5, Canada. ${ }^{7}$ The George Institute for Global Health, Imperial College London, London, UK.

Received: 25 June 2020 Accepted: 17 January 2021

Published online: 28 January 2021

\section{References}

1. Ahmed SQ, Liu LL, Tsui AO. Maternal deaths averted by contraceptive use: an analysis of 172 countries. Lancet. 2012;380(9837):111-25.

2. WHO. Contraception. Fact sheet. 2014. Availablfe from: https://apps.who.int/ iris/bitstream/handle/10665/112319/WHO_RHR_14.07_eng.pdf?ua=1. [Accessed on august 24, 2020].

3. Bongaarts J, Cleland J, Townsend JW, Bertrand JT, Gupta MD. Family planning programs for the 21st century. New York: Population Council; 2012.

4. WHO. Family planning/contraception methods. 2020. Available from: https://www.who.int/news-room/fact-sheets/detail/family-planningcontraception $\geq \leq$ http://www.who.int/news-room/fact-sheets/detail/familyplanning-contraception\%3e. [Accessed on August 05, 2020].

5. United Nations. Department of Economic and Social Affairs, Population Division (2017): World Family Planning; 2017. Highlights (ST/ESA/SER.A/414). 2017

6. Endriyas M, Eshete A, Mekonnen E, Misganaw T, Shiferaw M, Ayele S. Contraceptive utilization and associated factors among women of reproductive age group in southern nations nationalities and peoples' region, Ethiopia: cross-sectional survey, mixed-methods. Contraception Reprod Med. 2017;2:10. https://doi.org/10.1186/s40834-016-0036-z. 
7. Agence Nationale de la Statistique et de la Démographie (ANSD) [Senegal], and ICF International. Enquête Démographique et de Santé à Indicateurs Multiples au Sénégal (EDS-MICS) 2010-2011. Calverton, Maryland, USA: ANSD and ICF International; 2012

8. United Nations. Department of Economic and Social Affairs, population division (2017b). Model-based estimates and projections of family planning indicators. 2017. New York: United Nations; 2017.

9. USAID. West Africa: Senegal [Available from: http://www.watradehub.com/ en/countries/senegal/.[ Accessed on April 10, 2020].

10. Agence Nationale de la Statistique et de la Démographie ANSD/Sénégal and ICF. Sénégal: Enquête Démographique et de Santé Continue (EDS Continue 2015). Rockville, Maryland, USA: ANSD/Senegal and ICF; 2016.

11. Dulitha F, Nalika G, Upul S, Chrishantha WM, De Alwis SR, Hemantha S, et al. Risk factors for teenage pregnancies in Sri Lanka: perspective of a community based study. 2013;7(4):269-84.

12. Wuni C, Turpin CA, Dassah ET. Determinants of contraceptive use and future contraceptive intentions of women attending child welfare clinics in urban Ghana. BMC Public Health. 2018;18:79. https://doi.org/10.1186/s12889017-4641-9.

13. Cronin CJ, Guilkey DK, Speizer IS. The effect of health facility access and quality on family planning decisions in urban Senegal. Health Economic. 2017;27(3):576-91.

14. Stephenson R, Baschieri A, Clements S, Hennick M, Madise N. Contextual influences on modern contraceptive use in sub-Saharan Africa. Am J Public Health. 2007;97(7):1233-40

15. Kamal SM. Socioeconomic factors associated with contraceptive use and method choice in urban slums of Bangladesh. Asia Pac J Public Health. 2015;27(2):2661-76 https:/doi.org/.

16. Ajong AB, Njotang PN, Yakum MN, Essi MJ, Essiben F, Eko FEE, et al. Determinants of unmet need for family planning among women in urban Cameroon : a cross sectional survey in the Biyem-Assi Health District. Yaoundé BMC Womens Health. 2016;16(4):1-8.

17. Cavallaro FL, Benova L, Macleod D, Faye A, Lynch CA. Examining trends in family planning among harder-to-reach women in Senegal 1992-2014. Sci Rep. 2017;7:41006.

18. Yaya S, Uthman OA, Ekholuenetale M, Ghose BG. Women empowerment as an enabling factor of contraceptive use in sub-Saharan Africa: a multilevel analysis of cross-sectional surveys of 32 countries. Reprod Health. 2018;15: 214. https://doi.org/10.1186/s12978-018-0658-5 z.

19. Ahinkorah BO. Predictors of unmet need for contraceptionamong adolescent girls and young womenin selected high fertility countriesin subSaharan Africa: A multilevel mixed effects analysis. PLoS One. 2020;15(8): e0236352. https://doi.org/10.1371/journal.pone.0236352.

20. Gubhaju B. The influence of wives' and husbands' education levels on contraceptive method choice in Nepal,1996-2006. Int Perspect Sex Reprod Health. 2009;35(4):176-85.

21. Hossain M, Ahmed S, Rogers L. Does a wife's education influence spousal agreement on approval of family planning? Random effects modeling using data from two west African countries. J Health Care Poor Underserved. 2014;25:562-76. https://doi.org/10.1353/hpu.2014.0098.

22. Richard ML, Paul K, Anne-Marie N. Influence of mass media on family planning methods use among couples in Gashenyi Sector Rwanda. Int J Multidisciplinary Res Dev. 2017;4(6):336-43.

23. Howard-Grabman L, Snetro G. How to mobilize communities for health and social change. Baltimore: Health Communication Partnership; 2003. Available at http://www.jhuccp.org/hcp/pubs/tools/HowtoMobilize/ind> ex.html. Accessed on 8 April 2020

24. Kipping R, Campbell RM, MacArthur G, Gunnell D, MJJoPH H. Multiple risk behaviour in adolescence. J Public Health (Oxf). 2012;34(suppl_1):i1-2.

25. Ub L. Realising the health and wellbeing of adolescents. BMJ. 2015;351:h4119.

26. Currie J. Healthy, wealthy, and wise: socioeconomic status, poor health in childhood, and human capital development. J Econ Lit, Am Econ Assoc. 2009:47(1):87-122.

27. Nalwadda G, Mirembe F, Byamugisha J, Faxelid E. Persistent high fertility in Uganda: young people recount obstacles and enabling factors to use of contraceptives. BMC Public Health. 2010;10(1):1-13.

28. Mostafa KIA. Contraceptive use: socioeconomic correlates and method choices in rural Bangladesh. Asia Pac J Public Health. 2010;22(4):436-50.

29. Solanke BL. Socio-demographic factors associated with unmet need for family planning among women who experienced pregnancy termination in Nigeria. Afr J Psychol Study Soc Issues. 2016;19(3):112-25.
30. Das JK, Salam RA, Thornburg KL, Prentice AM, Campisi S, Lassi ZS, et al. Nutrition in adolescents: physiology, metabolism, and nutritional needs. Ann N Y Acad Sci. 2017;1393(1):21-33.

31. Ritchwood TD, Ford H, DeCoster J, Sutton M, JEJC L. Risky sexual behavior and substance use among adolescents: A meta-analysis. Child Youth Serv Rev. 2015;52:74-88.

32. Cunningham K, Martinez DA, Scott-Sheldon LA, Carey KB, MPJJoc C. Alcohol use and sexual risk behaviors among adolescents with psychiatric disorders: A systematic review and meta-analysis. J Child Adolesc Subst Abuse. 2017; 26(5):353-66.

33. Obasohan PE. Religion, Ethnicity and Contraceptive Use among Reproductive age Women in Nigeria. Int J MCH AIDS. 2015;3(1):63-73.

34. Dialogue WFD. Understanding religious influences on family planning. Finding from Monitoring and Evaluation in Senegal. Washington, DC.: World Faiths Development Dialogue; 2017.

35. Assaf $S$, Wang W, Mallick L. Quality of care in family planning services in Senegal and their outcomes. BMC Health Serv Res. 2017;17:346.

36. Sidibe AM, Kadetz PI, Hesketh T. Factors impacting family planning use in Mali and Senegal. Int J Environ Res Public Health. 2020;17:4399. https://doi. org/10.3390/ijerph17124399.

37. Isa Al, Gani IOOJOJoO, Gynecology. Socio-demographic determinants of teenage pregnancy in the Niger Delta of Nigeria 2012;2(03):239.

38. UNICEF. Young people and family planning: teenage pregnancy. New York: UNICEF; 2008.

39. Adu-Gyamfi E. Assessing the effect of teenage pregnancy on achieving universal basic education in Ghana: a case study of upper Denkyira West District. J Educ Pract. 2014;5(17):46-60.

40. Ayanaw Habitu Y, Yalew A, TJJop AB. Prevalence and Factors Associated with Teenage Pregnancy, Northeast Ethiopia, 2017: A Cross-Sectional Study. J Pregnancy. 2018;2018:1714527.

41. Nyarko SHJJJS. Determinants of adolescent fertility in Ghana. Int J Sci. 2012; 5(1):21-32.

42. Ministère de la Santé et de l'Action Sociale. Pyramide de Santé. Available from: http://www.sante.gouv.sn/page-reader-contentdetails.php?.jpage= $\mathrm{NTg}=\& \mathrm{jmenu}=\mathrm{Mg}==$. [Accessed on: Sep 17, 2020].

43. Darroch JE, Woog V, Bankole A. Ashford LS. Guttmacher Institute: Costs and benefits of meeting the contraceptive needs of adolescents; 2016.

44. Sedgh G, Finer LB, Bankole A, Eilers MA, Singh S. Adolescent pregnancy, birth, and abortion rates across countries: levels and recent trends. J Adolesc Health. 2015:56(2):223-30.

45. Agence Nationale de la Statistique et de la Démographie (ANSD) [Senegal], and ICF International. Enquête Démographique et de Santé à Indicateurs Multiples au Sénégal (EDS-MICS) 2016-2017. Calverton, Maryland, USA: ANSD and ICF International; 2017

46. Bradley SEK, Croft TN, Fishel JD, Westoff CF. Revising Unmet Need for Family Planning. DHS Analytical Studies No. 25 [available at $\leq$ https://dhsprogram. com/pubs/pdf/AS25/AS25\%5B12June2012\%5D.pdf $\geq$. Accessed on 8 April 2020].

47. Mohammed A, Woldeyohannes D, Feleke A, Megabiaw B. Determinants of modern contraceptive utilization among married women of reproductive age group in north Shoa zone, Amhara region, Ethiopia. Reprod Health. 2014;11(1):13.

48. Hailu TG. Determinants and cross-regional variations of contraceptive prevalence rate in Ethiopia: a multilevel modeling approach. Am J Math Stat. 2015:5(3):95-110.

49. Prata N, Bell S, Weidert K, Nieto-Andrade B, Carvalho A, Neves I. Varying family planning strategies across age categories: differences in factors associated with current modern contraceptive use among youth and adult women in Luanda, Angola. Open Access J Contraception. 2016;7:1.

50. Ahinkorah BO. Predictors of unmet need for contraception among adolescent girls and young women in selected high fertility countries in sub-Saharan Africa: a multilevel mixed effects analysis. PLoS One. 2020;15(8):e0236352.

51. Kishor S, Subaiya L. Understanding Women's empowerment: a comparative analysis of demographic and health surveys (DHS) data. DHS comparative reports no. 20. Calverton, Maryland, USA: Macro International Inc;; 2008. https:// dhsprogram.com/publications/publication-cr20-comparativereports.ffm

52. Guide to DHS Statistics. Indicators of Women empowerment. [Available at < https://dhsprogram.com/data/Guide-to-DHS

53. Austin PC, Merlo J. Intermediate and advanced topics in multilevel logistic regression analysis. Stat Med. 2017;36(20):3257-77. https://doi.org/10.1002/ sim.7336 PMID: 28543517. 
54. Gelman A, Hill J. Data analysis using regression and multilevelhierarchical models: Cambridge University press New York. NY: USA; 2007.

55. Cherry AL, Dillon ME. International handbook of adolescent pregnancy: medical, psychosocial, and public health responses. New York: Springer Science \& Business Media; 2014.

56. Daoud Jl. Multicollinearity and regression analysis: IOP Conf. Series: Journal of Physics: Conf. Series. 2017;949:1-7.

57. O'brien RM. A caution regarding rules of thumb for variance inflation factors. Qual Quant. 2007:41(5):673-90.

58. DHS Program Sampling weighting. [Available from: https://blog. dhsprogram.com/sampling-weighting-at-dhs/>. Accessed on 8 April 2020.

59. Darroch JE et al., Adding It Up: Costs and Benefits of Meeting the Contraceptive Needs of Adolescents, New York: Guttmacher Institute; 2016, https://www.guttmacher.org/report/adding-it-meeting-contraceptive-needsof-adolescents.

60. Daff BM, Seck C, Belkhayat H, Sutton P. Informed push distribution of contraceptives in Senegal reduces stockouts and improves quality of family planning services. Global Health. 2014:245-52. https://doi.org/10.9745/ghspd-13-00171.

61. Neal S, Matthews Z, Frost M, Fogstad H, Camacho AV, Laski L. Childbearing in adolescents aged 12-15 years in low resource countries: a neglected issue. New estimates from demographic and household surveys in 42 countries. Acta Obstet Gynecol Scand. 2012;91(9):1114-8.

62. UNICEF. The state of the world's children 2011: adolescence an age of opportunity. New York: UNICEF; 2013.

\section{Publisher's Note}

Springer Nature remains neutral with regard to jurisdictional claims in published maps and institutional affiliations.

Ready to submit your research? Choose BMC and benefit from:

- fast, convenient online submission

- thorough peer review by experienced researchers in your field

- rapid publication on acceptance

- support for research data, including large and complex data types

- gold Open Access which fosters wider collaboration and increased citations

- maximum visibility for your research: over $100 \mathrm{M}$ website views per year

At $\mathrm{BMC}$, research is always in progress.

Learn more biomedcentral.com/submissions 